Research Paper

\title{
Genetic Polymorphisms in SLC23A2 as Predictive Biomarkers of Severe Acute Toxicities after Treatment with a Definitive 5-Fluorouracil/Cisplatin-Based Chemoradiotherapy in Japanese Patients with Esophageal Squamous Cell Carcinoma
}

Tetsuya Minegaki 1, Akiko Kuwahara 2,3, Motohiro Yamamori 2,3, Tsutomu Nakamura 2, Tatsuya Okuno 2, Ikuya Miki 2, Hideaki Omatsu 2, Takao Tamura 2,4, Midori Hirai 2, Takeshi Azuma 2, Toshiyuki Sakaeda 2,5凶 and Kohshi Nishiguchi ${ }^{1,2}{ }^{\bowtie}$

1. Faculty of Pharmaceutical Sciences, Kyoto Pharmaceutical University, Kyoto 607-8414, Japan.

2. Kobe University Graduate School of Medicine, Kobe 650-0017, Japan.

3. School of Pharmacy and Pharmaceutical Sciences, Mukogawa Women's University, Nishinomiya 663-8179, Japan.

4. Department of Medical Oncology, Nara Hospital, Kinki University Faculty of Medicine, Nara 630-0293, Japan.

5. Graduate School of Pharmaceutical Sciences, Kyoto University, Kyoto 606-8501, Japan.

$\triangle$ Corresponding authors: Toshiyuki Sakaeda, Ph.D., Graduate School of Pharmaceutical Sciences, Kyoto University, Kyoto 606-8501, Japan. Tel: +81-75-753-9560, Fax: +81-75-753-4502, E-Mail: sakaedat@pharm.kyoto-u.ac.jp; Kohshi Nishiguchi, Ph.D., Faculty of Pharmaceutical Sciences, Kyoto Pharmaceutical University, Kyoto 607-8414, Japan. Tel: +81-75-595-4627, Fax: +81-75-595-4752, E-Mail: kohshi@mb.kyoto-phu.ac.jp.

(c) Ivyspring International Publisher. This is an open-access article distributed under the terms of the Creative Commons License (http://creativecommons.org/ licenses/by-nc-nd/3.0/). Reproduction is permitted for personal, noncommercial use, provided that the article is in whole, unmodified, and properly cited.

Received: 2013.09.13; Accepted: 2014.01.22; Published: 2014.02.07

\begin{abstract}
Objective: Definitive chemoradiotherapy (CRT) with 5-fluorouracil (5-FU) and cisplatin (CDDP) is one of the standard therapies for esophageal squamous cell carcinoma (ESCC); however, inter-individual variations in clinical outcomes have yet to be investigated. In the present study, single nucleotide polymorphisms (SNPs) in SLC23A2 gene were retrospectively evaluated in 49 japanese patients with ESCC who were treated with a definitive 5-FU/CDDP-based CRT, and the predictive values for the clinical response, severe acute toxicities, and long-term survival were assessed.

Methods: A course consisted of the continuous infusion of $5-\mathrm{FU}$ at $400 \mathrm{mg} / \mathrm{m}^{2} /$ day for days I-5 and $8-12$, the infusion of CDDP at $40 \mathrm{mg} / \mathrm{m}^{2} /$ day on days I and 8 , and radiation at $2 \mathrm{~Gy} /$ day on days I to 5,8 to 12 , and 15 to 19 , with a second course being repeated after a 2-week interval. The SLC23A2 SNPs rs268I I 16, rs 13037458, rs 1715364, rs4987219, and rs I I 0277 were evaluated.

Results: The rs 268 I I I6 and rs 13037458 had a tendency to predict the clinical response $(p=0.144$ and 0.085 , respectively) and long-term survival $(p=0.142$ and 0.056 , respectively). The rs 4987219 and rsIII 0277 correlated with severe acute leukopenia $(p=0.025)$ and stomatitis $(p=0.019)$, respectively.

Conclusions: Further investigations with a larger number of patients or an in vitro study are needed to confirm the predictive values of genetic polymorphisms in SLC23A2.
\end{abstract}

Key words: esophageal squamous cell carcinoma, chemoradiotherapy, biomarker, SLC23A2, polymorphism.

\section{Introduction}

A definitive chemoradiotherapy (CRT) with 5-fluorouracil (5-FU) and cisplatin (CDDP) was proposed for the treatment of advanced metastatic esophageal squamous cell carcinoma (ESCC) in the late 1990s, and two independent clinical investigations have shown the curative potential for unresec- 
table ESCC with T4 or M1a [1,2]. A long-term evaluation of efficacy and toxicity with 139 patients resulted in a complete response (CR) rate of $56 \%$, along with a 5-year survival rate of $29 \%$ [3-5]. However, treatment-related toxicities were severe, and life-threatening late toxicities may have occurred [4, 5]. A series of studies was performed to identify the predictive biomarkers of the clinical response 1 month after this definitive 5-FU/CDDP-based CRT or severe acute toxicities this treatment in Japanese patients with ESCC, including genetic polymorphisms and the plasma concentrations of 5-FU [6-10]. Their effects on long-term survival and/or late toxicities were also evaluated [11, 12]; however, these studies did not always result in a sufficient statistical power to be predictive.

In this study, we focused on vitamin $C$ transporters (SVCTs). Vitamin C is an essential cofactor in various enzymatic hydroxylation reactions and is known to be the antioxidant that prevents carcinogenesis-inducing oxidative damage [13, 14]. Tsukaguchi et al. cloned 2 sodium-dependent SVCTs, SVCT1 and SVCT2 in 1999, which are encoded by SLC23A1 and SLC23A2, respectively [15]. They also demonstrated that these transporters facilitated uptake of the reduced form of vitamin C into cells. SVCT1 is mainly expressed in epithelial tissues (the intestine, liver, and kidney) and plays a key role in the intestinal absorption and renal re-absorption of vitamin C. SVCT2 was shown to be widely expressed throughout the body and is necessary for the maintenance of cellular oxidation-reduction states [15-18]. Single nucleotide polymorphisms (SNPs) have recently been identified in these transporters. Several clinical studies were conducted to identify associations between SLC23A2 SNPs and the risk of cancers such as lymphoma [19], HPV16-associated head and neck cancer [20], colorectal cancer [21], and gastric cancer [22]. Moreover, Abdel-Latif et al. reported that vitamin $\mathrm{C}$ enhanced 5-FU and CDDP sensitization in esophageal cancer cells in vitro [23]. The results of cDNA microarray analysis, conducted by Karasawa et al., indicated that 5-FU-resistant colon cancer cells expressed lower levels of SLC23A2 mRNA than parental cells [24]. We recently reported a strong correlation between SLC23A2 mRNA levels and 5-FU sensitivity in esophageal carcinoma cell lines [25]. Taken together, we speculated that SLC23A2 SNPs could be predictive factors of clinical outcomes, especially of the clinical response and long-term survival, following treatment with 5-FU or CDDP.

In the present study, SLC23A2 SNPs were retrospectively evaluated in Japanese patients with ESCC who underwent a definitive 5-FU/CDDP-based
CRT, and their associations with the clinical response, severe acute toxicities, and long-term survival were analyzed. The subjects in this study were the same as those in our previous reports [10-12].

\section{Methods}

\section{Ethics statements}

A series of studies has been performed to find the predictive biomarkers of the clinical response, severe acute toxicities and long-term survival following treatment with a definitive 5-FU/CDDP-based CRT in Japanese patients with ESCC [6-12]. These studies were conducted with the authorization of the Institutional Review Board (IRB) and followed the medical research council guidelines of Kobe University. All patients analyzed agreed to these studies and the preservation of genomic DNA for future investigations. Additional studies were again authorized by the IRB and followed the guidelines of Kobe University. Written informed consent was obtained from all participants prior to genotyping.

\section{Patients}

The subjects were recruited from a database of patients diagnosed with ESCC and treated with a definitive 5-FU/CDDP-based CRT at Kobe University Hospital between 2003 and 2006. Throughout this period, the treatment regimen was standardized at our institution as described below, and was employed for those who were medically unfit for an operation or refused an operation.

In the present study, 49 patients analyzed were on the basis of the following criteria: 1) clinical stage $\mathrm{T} 1$ to T4, N0 or N1, and M0 or M1 according to the International Union Against Cancer tumor-node-metastasis (TNM) classification; 2) aged less than 85 years; 3) an Eastern Cooperative Oncology Group performance status of 0 to 2; 4) adequate bone marrow, renal, and hepatic function; 5) no prior chemotherapy; 6) no severe medical complications; 7) no other active malignancies (except for early cancer) and 8) availability of written informed consent, as described above. The tumors were histologically confirmed to be primary, and no patients with recurrence were included in this study.

\section{Protocol}

A course consisted of the continuous infusion of 5 -FU at $400 \mathrm{mg} / \mathrm{m}^{2} /$ day for days $1-5$ and $8-12$, the infusion of CDDP at $40 \mathrm{mg} / \mathrm{m}^{2} /$ day on days 1 and 8 , and the radiation at $2 \mathrm{~Gy} /$ day on days 1-5, 8-12, and 15-19, with a second course being repeated after a 2 -week interval $[1,2]$. When detecting grade $3 / 4$ hematological toxicities, chemotherapy was withheld until the blood cell counts recovered beyond the crit- 
ical level, and then a reduced dose was resumed. For fever higher than grade 2, chemotherapy was withheld pending improvement. For renal toxicity, the subsequent dose of CDDP was reduced depending on the degree of toxicity. Radiotherapy was also withheld during the time when the chemotherapy was withheld for severe hematological and/or non-hematological toxicities. In principle, the total radiation dose was not reduced.

\section{SNP selection}

SLC23A2 SNPs candidates were selected according to the following criteria. First, there were reports showing associations with the risk of cancer. Second, minor allelic SNP frequencies had to be more than $40 \%$ as per the National Center for Biotechnology Information (NCBI) variation database (dbSNP). Allelic frequencies were related to genetic variations in the SNPs studied, and reference sequences were adopted on the basis of information from the NCBI dbSNP database. A threshold value of $40 \%$ was set by referring to the rate of CR $(46.9 \%)$ or severe acute leukopenia (42.9\%). Five SNPs, rs2681116 in intron 2, rs13037458 in intron 2, rs1715364 in intron 3, rs4987219 in intron 8, and rs1110277 in exon 11 (synonymous), met the criteria above.

\section{Genotyping}

To analyze rs13037458, rs1715364, rs4987219, and rs1110277, genomic DNA was isolated from whole blood using a TaqMan ${ }^{\circledR}$ Sample-to-SNPTM kit (Applied Biosystems, Foster City, CA, USA), according to the manufacturer's instructions. SNPs were evaluated using TaqMan ${ }^{\circledR}$ MGB probe-based polymerase chain reaction (PCR) and the StepOne ${ }^{\mathrm{TM}}$ real-time PCR system (Applied Biosystems). For rs2681116, genomic DNA was extracted from whole blood using a QIAamp DNA blood mini kit (QIAGEN, Tokyo, Japan), and DNA direct sequencing was performed. Briefly, PCR was performed for the sequence containing rs2681116 using the hot start version of Takara EX Taq $^{\circledR}$ (TAKARA BIO, Shiga, Japan) and GeneAmp ${ }^{\circledR}$ PCR system 9700 (Applied Biosystems). The forward and reverse primer sequences for the PCR reaction were 5'-CCAGTTGTGTTTCCTTTTCCTTTTCT- $3^{\prime}$ and 5'-GTAGAAGGATCACATAAGCCCAGTAG-3', respectively. Reactions were performed at $94^{\circ} \mathrm{C}$ for 3 min, followed by 35 cycles of $94^{\circ} \mathrm{C}$ for $20 \mathrm{~s}, 61^{\circ} \mathrm{C}$ for 20 $\mathrm{s}$, and $72^{\circ} \mathrm{C}$ for $30 \mathrm{~s}$, and finally at $72^{\circ} \mathrm{C}$ for $5 \mathrm{~min}$ with a cooling step at $4^{\circ} \mathrm{C}$. PCR products (131 bp) were subjected to electrophoresis on 3\% agarose gels. After clear bands of the expected sizes were observed, the products were used as templates for sequencing reactions along with the BigDye ${ }^{\circledR}$ Terminator v1.1 cycle sequencing kit (Applied Biosystems); these were pu- rified using the BigDye ${ }^{\circledR}$ XTerminator ${ }^{\mathrm{TM}}$ purification kit (Applied Biosystems). DNA sequencing analysis was performed on an ABI PRISM ${ }^{\circledR} 310$ genetic analyzer (Applied Biosystems) with sequencing analysis $^{\mathrm{TM}}$ software, ver. 3.7 (Applied Biosystems).

\section{Severe acute toxicities}

This definitive 5-FU/CDDP-based CRT is associated with acute toxicities, predominantly leukopenia, stomatitis, and cheilitis [1-5]. Toxicity was evaluated using criteria defined by the Japan Clinical Oncology Group. These criteria were based on the National Cancer Institute Common Toxicity Criteria. Toxicity was assessed on a 2 to 3 day basis during the CRT and subsequent hospitalization period and on every visit after the completion of CRT. Episodes of leukopenia, stomatitis, and cheilitis during the first 2 courses and subsequent 2 weeks (until day 70) were recorded as acute toxicities and those of grade 3 or more as severe acute toxicities.

\section{Clinical response}

The clinical response was evaluated as reported previously [1-5]. Briefly, a complete response (CR) was defined as the complete disappearance of all measurable and assessable disease at the first evaluation, which was performed 1 month after the completion of CRT to determine whether the disease had progressed. The clinical response was evaluated by endoscopy and chest and abdominal computed tomography (CT) scans in each course. A CR at the primary site was evaluated by endoscopic examination when all of the following criteria were satisfied on observation of the entire esophagus: 1) disappearance of the tumor lesion; 2) disappearance of ulceration (slough); and 3) absence of cancer cells in biopsy specimens. If small nodes of $1 \mathrm{~cm}$ or less were detected on CT scans, the recovery was defined as an "uncertain CR" after confirmation of no progression for at least 3 months. An "uncertain CR" was included as a CR when calculating the CR rate. When these criteria were not satisfied, a non-CR was assigned. The existence of erosion, a granular protruded lesion, an ulcer scar, and $1.2 \mathrm{w} / \mathrm{v} \%$ iodine/glycerin-voiding lesions did not prevent an evaluation of CR. The evaluations were performed every month for the first 3 months, and when the criteria for CR were not satisfied at 3 months, the result was changed to non-CR. Follow-up evaluations were performed thereafter every 3 months for 3 years by endoscopy and CT scan. After 3 years, patients were seen every 6 months. During the follow-up period, a routine course of physical examinations and clinical laboratory tests was performed to check the patient's health. 


\section{Survival}

Survival time was defined as the time from treatment initiation to death from any cause or to the last date of confirmation of survival. Survival data were updated on June 25, 2011.

\section{Data analysis and statistics}

All values reported are the mean \pm standard deviation (SD) without any explanatory notes. Fisher's exact test was used for the analysis of contingency tables. The differences between overall survival curves were analyzed using the log-rank test. $P$ values of less than 0.05 (two-tailed) were considered significant.

\section{Results}

A total of 49 patients were analyzed, consisting of 46 males and 3 females. The average age was $64.5 \pm 7.4$ years. The ratio of $\mathrm{T} 1 / \mathrm{T} 2 / \mathrm{T} 3 / \mathrm{T} 4$ was 16/6/15/12 with those of N0/N1 and M0/M1 being $22 / 27$ and $41 / 8$, respectively, resulting in stage I/II/III/IV of $12 / 10 / 19 / 8$. The CR and 5 -year survival rates were $46.9 \%(23 / 49)$ and $42.9 \%$ (21/49), respectively. Severe acute leukopenia, stomatitis, and cheilitis occurred in $42.9 \%$ (21/49), $14.3 \%$ (7/49), and $16.3 \%(8 / 49)$ of cases, respectively.

Table 1 summarizes the data on associations between 5 SLC23A2 SNPs and the clinical response. No significant correlations were observed between the genotypes examined and the clinical response; however, rs2681116 $(\mathrm{p}=0.144)$ and $\mathrm{rs} 13037458$ $(p=0.085)$ had a tendency to associate with. As shown in Figure 1, these 2 SNPs also showed a tendency to predict long-term survival ( $\mathrm{p}=0.142$ for $\mathrm{rs} 2681116$, $\mathrm{p}=0.056$ for rs13037458). Table 2 lists associations with severe acute leukopenia, stomatitis, and cheilitis. rs4987219 and rs1110277 significantly correlated with leukopenia $(p=0.025)$ and stomatitis $(p=0.019)$, respectively.

Table I. Association between SLC23A2 genotypes and the clinical response in 49 patients after treatment with a definitive 5 -fluorouracil/cisplatin-based chemoradiotherapy.

\begin{tabular}{llllll}
\hline & & Total & CR & non-CR & p value \\
\hline rs2681116 & TT & 9 & 7 & 2 & 0.144 \\
& TC & 27 & 11 & 16 & \\
& CC & 13 & 5 & 8 & \\
rs13037458 & AA & 12 & 9 & 3 & 0.085 \\
& AC & 31 & 12 & 19 & \\
& CC & 6 & 2 & 4 & \\
rs1715364 & TT & 4 & 1 & 3 & 0.758 \\
& TC & 25 & 12 & 13 & \\
& CC & 20 & 10 & 10 & \\
rs4987219 & GG & 2 & 1 & 1 & 0.480 \\
& GC & 15 & 5 & 10 & \\
& CC & 32 & 17 & 15 & \\
rs1110277 & GG & 2 & 0 & 2 & 0.438 \\
& GA & 19 & 8 & 11 & \\
& AA & 28 & 15 & 13 & \\
\hline
\end{tabular}

The rate of the complete response (CR) was $46.9 \%$ (23/49). Frequencies in patients with $\mathrm{CR}$ were compared with those with non-CR. Significance was assessed using

Fisher's exact test.

Table 2. Association between SLC23A2 genotypes and severe acute leukopenia, stomatitis, and cheilitis in 49 patients after treatment with a definitive 5-fluorouracil/cisplatin-based chemoradiotherapy.

\begin{tabular}{|c|c|c|c|c|c|c|c|c|c|c|}
\hline & & \multicolumn{3}{|c|}{ Leukopenia } & \multicolumn{3}{|c|}{ Stomatitis } & \multicolumn{3}{|c|}{ Cheilitis } \\
\hline & & severe & not severe & $\mathrm{p}$ & severe & not severe & $\mathrm{p}$ & severe & not severe & $\mathrm{p}$ \\
\hline \multirow[t]{3}{*}{ rs2681116 } & $\mathrm{TT}$ & 4 & 5 & 0.540 & 2 & 7 & 0.291 & 1 & 8 & 0.250 \\
\hline & $\mathrm{TC}$ & 13 & 14 & & 2 & 25 & & 3 & 24 & \\
\hline & $\mathrm{CC}$ & 4 & 9 & & 3 & 10 & & 4 & 9 & \\
\hline \multirow[t]{3}{*}{ rs13037458 } & AA & 6 & 6 & 0.446 & 3 & 9 & 0.399 & 1 & 11 & 0.070 \\
\hline & $\mathrm{AC}$ & 14 & 17 & & 3 & 28 & & 4 & 27 & \\
\hline & $\mathrm{CC}$ & 1 & 5 & & 1 & 5 & & 3 & 3 & \\
\hline \multirow[t]{3}{*}{ rs1715364 } & TT & 1 & 3 & 0.123 & 0 & 4 & 0.832 & 0 & 4 & 0.344 \\
\hline & TC & 8 & 17 & & 3 & 22 & & 6 & 19 & \\
\hline & $\mathrm{CC}$ & 12 & 8 & & 4 & 16 & & 2 & 18 & \\
\hline \multirow[t]{3}{*}{ rs4987219 } & GG & 2 & 0 & 0.025 & 1 & 1 & 0.168 & 1 & 1 & 0.105 \\
\hline & GC & 3 & 12 & & 3 & 12 & & 4 & 11 & \\
\hline & $\mathrm{CC}$ & 16 & 16 & & 3 & 29 & & 3 & 29 & \\
\hline \multirow[t]{3}{*}{ rs1110277 } & GG & 0 & 2 & 0.612 & 2 & 0 & 0.019 & 1 & 1 & 0.062 \\
\hline & GA & 9 & 10 & & 2 & 17 & & 5 & 14 & \\
\hline & AA & 12 & 16 & & 3 & 25 & & 2 & 26 & \\
\hline
\end{tabular}

The rates of severe acute leukopenia, stomatitis, and cheilitis were $42.9 \%(21 / 49), 14.3 \%(7 / 49)$ and $16.3 \%(8 / 49)$, respectively. Frequencies in patients with these toxicities were compared with those without. Significance was assessed using Fisher's exact test. 
A) $\mathrm{rs} 2681116$

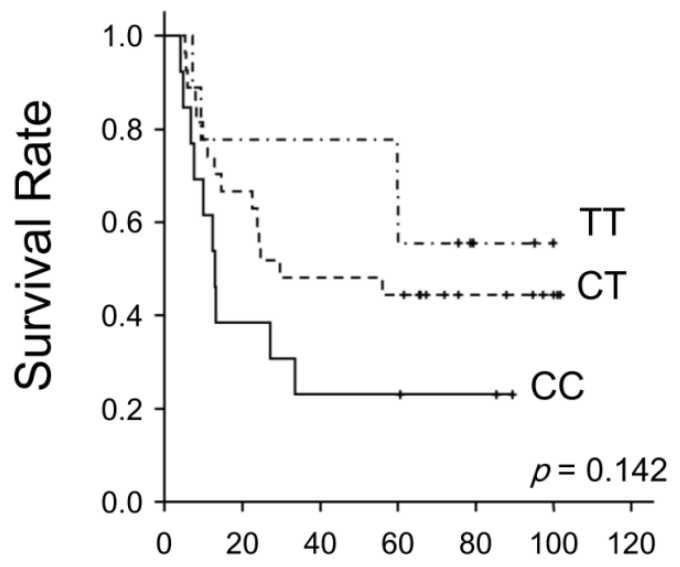

B) rs13037458

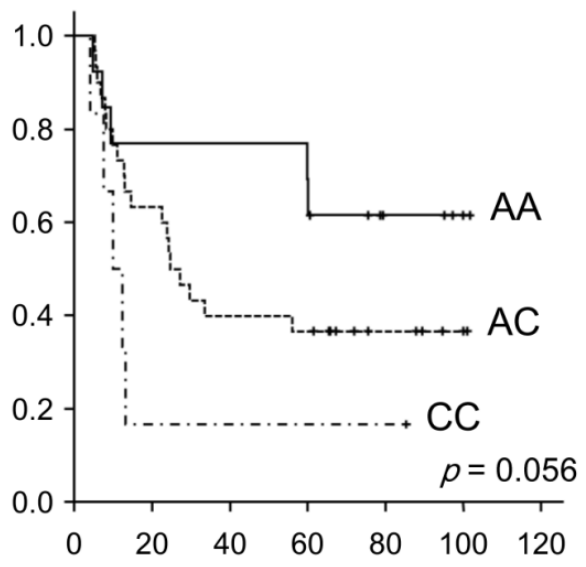

Survival Time (months)

Figure I. Association between the SLC23A2 genotypes of rs268III6 and rs 13037458 and long-term survival in 49 patients following treatment with a definitive 5-fluorouracil/cisplatin-based chemoradiotherapy. Long-term survival was possibly associated with these genotypes ( $\mathrm{F}=0 . \mathrm{I} 42$ for $\mathrm{rs} 268 \mathrm{I}$ I 6 and $\mathrm{p}=0.056$ for rs I 3037458; the log-rank test).

\section{Discussion}

To the best of our knowledge, this is the first study to investigate associations between genetic polymorphisms in SLC23A2 and the clinical outcomes of definitive 5-FU/CDDP-based CRT. There was a tendency for rs2681116 in intron 2 and rs13037458 in intron 2 to predict the clinical response (Table 1). The clinical response was significantly correlated with long-term survival in the same patients [11]; and therefore, they also had a tendency to predict the long-term survival (Fig. 1). The accumulation of cellular vitamin $\mathrm{C}$, and perhaps 5-FU, may have been altered, resulting in an alteration in cellular sensitivity to 5-FU and CDDP. Unfortunately, no significant change was observed in the clinical response or long-term survival, which can be explained by the small sample size of the present study.

SVCT2 is known to maintain the cellular redox state through the uptake of vitamin $\mathrm{C}$ into the cell [17]. Chemotherapy-induced normal tissue toxicities have been related to the cellular redox balance [26]. Numazawa et al. [27] suggested that 5-FU-induced myelosuppression was involved in oxidative stress. Additionally, García-de-la-Asunción et al. [28] reported that combined treatment with vitamin $C$ and vitamin $E$ decreased the rate of leukopenia induced by 3'-azido-2',3'-dideoxythimidine (AZT), a therapeutic drug for acquired immunodeficiency syndrome, in mice. The same study indicated that oxidative stress was associated with the side effects of AZT [29-31]. In the present study, we showed that rs4987219 in intron 8 and the synonymous rs1110277 in exon 11 were associated with the severe acute leukopenia and stoma- titis, respectively. In 2004, the genomic structure and variations in human SLC23A1 and SLC23A2 gene was clarified by Eck et al. [32]. SLC23A2 gene was found to be 158,398 bp long, containing 17 exons, but several large introns resulted in transcript of 7,421 bp [32]. More than 40 variants, including rs4987219 and rs1110277, were found in SLC23A2 gene at that time, but the pattern of linkage disequilibrium across the gene could not be clarified due to large size [32]. It is not surprising that a genetic variant is linked with others and the effect on transport activity varies among variants; however, no associations of rs4987219 with stomatitis and rs1110277 with leukopenia suggested that the effect on transport activity is tissue-dependent.

\section{Conclusions}

The SLC23A2 SNPs rs2681116, rs13037458, rs1715364, rs4987219, and rs1110277 were assessed in terms of the predictability of clinical outcomes following treatment with a definitive 5-FU/CDDP-based CRT in Japanese patients with ESCC. The rs2681116 and rs13037458 had a tendency to predict the clinical response and long-term survival, while rs4987219 and rs1110277 were markers of severe acute leukopenia and stomatitis, respectively. Further investigations with a larger number of patients or an in vitro study are needed to confirm the predictive values of genetic polymorphisms in SLC23A2.

\section{Acknowledgments}

This work was supported in part by a Grant-in-Aid for Scientific Research from the Ministry 
of Education, Culture, Sports, Science and Technology of Japan.

\section{Competing interests}

The authors declare that no conflict of interest exists.

\section{References}

1. Ohtsu A, Boku N, Muro K, et al. Definitive chemoradiotherapy for T4 and/or M1 lymph node squamous cell carcinoma of the esophagus. J Clin Oncol. 1999; 17: 2915-2921.

2. Kaneko K, Ito H, Konishi K, et al. Definitive chemoradiotherapy for patients with malignant stricture due to $\mathrm{T} 3$ or $\mathrm{T} 4$ squamous cell carcinoma of the oesophagus. Br J Cancer. 2003; 88: 18-24.

3. Tahara M, Ohtsu A, Hironaka S, et al. Clinical impact of criteria for complete response (CR) of primary site to treatment of esophageal cancer. Jpn J Clin Oncol. 2005; 35: 316-323.

4. Ishikura S, Nihei $\mathrm{K}$, Ohtsu A, et al. Long-term toxicity after definitive chemoradiotherapy for squamous cell carcinoma of the thoracic esophagus. J Clin Oncol. 2003; 21: 2697-2702.

5. Kumekawa Y, Kaneko K, Ito H, et al. Late toxicity in complete response cases after definitive chemoradiotherapy for esophageal squamous cell carcinoma. J Gastroenterol. 2006; 41: 425-432.

6. Miki I, Tamura T, Nakamura T, et al. Circadian variability of pharmacokinetics of 5-fluorouracil and CLOCK T3111C genetic polymorphism in patients with esophageal carcinoma. Ther Drug Monit. 2005; 27: 369-374.

7. Okuno T, Tamura T, Yamamori M, et al. Favorable genetic polymorphisms predictive of clinical outcome of chemoradiotherapy for stage II/III esophageal squamous cell carcinoma in Japanese. Am J Clin Oncol. 2007; 30: 252-257.

8. Sakaeda T, Yamamori M, Kuwahara A, et al. VEGF G-1154A is predictive of severe acute toxicities during chemoradiotherapy for esophageal squamous cell carcinoma in Japanese patients. Ther Drug Monit. 2008; 30: 497-503.

9. Kuwahara A, Yamamori M, Fujita M, et al. TNFRSF1B A1466G genotype is predictive of clinical efficacy after treatment with a definitive 5-fluorouracil/cisplatin-based chemoradiotherapy in Japanese patients with esophageal squamous cell carcinoma. J Exp Clin Cancer Res. 2010; 29: 100.

10. Miki I, Nakamura T, Kuwahara A, et al. THRB genetic polymorphisms can predict severe myelotoxicity after definitive chemoradiotherapy in patients with esophageal squamous cell carcinoma. Int J Med Sci. 2012; 9: 748-756.

11. Kuwahara A, Yamamori M, Kadoyama K, et al. Effects of plasma concentrations of 5-fluorouracil on long-term survival after treatment with a definitive 5 -fluorouracil/cisplatin-based chemoradiotherapy in Japanese patients with esophageal squamous cell carcinoma. J Exp Clin Cancer Res. 2011; 30: 94.

12. Tamura T, Kuwahara A, Yamamori M, et al. VEGF-634C/G genotype is predictive of long-term survival after treatment with a definitive 5-fluorouracil/cisplatin-based chemoradiotherapy in Japanese patients with esophageal squamous cell carcinoma. Int J Med Sci. 2012; 9: 833-837.

13. Kryston TB, Georgiev AB, Pissis P, et al. Role of oxidative stress and DNA damage in human carcinogenesis. Mutat Res. 2011; 711: 193-201.

14. Byers T, Guerrero N. Epidemiologic evidence for vitamin $C$ and vitamin $E$ in cancer prevention. Am J Clin Nutr. 1995; 62: 1385S-1392S

15. Tsukaguchi $\mathrm{H}$, Tokui $\mathrm{T}$, Mackenzie $\mathrm{B}$, et al. A family of mammalian Na+-dependent L-ascorbic acid transporters. Nature. 1999; 399: 70-75.

16. Mandl J, Szarka A, Bánhegyi G. Vitamin C: update on physiology and pharmacology. Br J Pharmacol. 2009; 157: 1097-1110.

17. Savini I, Rossi A, Pierro C, et al. SVCT1 and SVCT2: key proteins for vitamin C uptake. Amino Acids. 2008; 34: 347-355.

18. Savini I, Rossi A, Catani MV, et al. Redox regulation of vitamin C transporter SVCT2 in C2C12 myotubes. Biochem Biophys Res Commun. 2007; 361: 385-390.

19. Skibola CF, Bracci PM, Halperin E, et al. Polymorphisms in the estrogen receptor 1 and vitamin $C$ and matrix metalloproteinase gene families are associated with susceptibility to lymphoma. PLoS One. 2008; 3: e2816.

20. Chen AA, Marsit CJ, Christensen BC, et al. Genetic variation in the vitamin C transporter, SLC23A2, modifies the risk of HPV16-associated head and neck cancer. Carcinogenesis. 2009; 30: 977-981.

21. Erichsen HC, Peters U, Eck P, et al. Genetic variation in sodium-dependent vitamin C transporters SLC23A1 and SLC23A2 and risk of advanced colorectal adenoma. Nutr Cancer. 2008; 60: 652-659.

22. Wright ME, Andreotti G, Lissowska J, et al. Genetic variation in sodium-dependent ascorbic acid transporters and risk of gastric cancer in Poland. Eur J Cancer. 2009; 45: 1824-1830.

23. Abdel-Latif MM, Raouf AA, Sabra K, et al. Vitamin C enhances chemosensitization of esophageal cancer cells in vitro. J Chemother. 2005; 17: 539-549.

24. Karasawa H, Miura K, Fujibuchi W, et al. Down-regulation of cIAP2 enhances 5-FU sensitivity through the apoptotic pathway in human colon cancer cells. Cancer Sci. 2009; 100: 903-913.

25. Minegaki T, Takara K, Hamaguchi R, et al. Factors affecting the sensitivity of human-derived esophageal carcinoma cell lines to 5-fluorouracil and cisplatin. Oncol Lett. 2013; 5: 427-434.
26. Doroshow JH. Redox modulation of chemotherapy-induced tumor cell killing and normal tissue toxicity. J Natl Cancer Inst. 2006; 98: 223-225.

27. Numazawa S, Sugihara K, Miyake S, et al. Possible involvement of oxidative stress in 5-fluorouracil-mediated myelosuppression in mice. Basic Clin Pharmacol Toxicol. 2011; 108: 40-45.

28. García-de-la-Asunción J, Gómez-Cambronero LG, Del Olmo ML, et al. Vitamins $\mathrm{C}$ and $\mathrm{E}$ prevent AZT-induced leukopenia and loss of cellularity in bone marrow. Studies in mice. Free Radic Res. 2007; 41: 330-334.

29. de la Asunción JG, del Olmo ML, Sastre J, et al. AZT treatment induces molecular and ultrastructural oxidative damage to muscle mitochondria. Prevention by antioxidant vitamins. J Clin Invest. 1998; 102: 4-9.

30. de la Asunción JG, del Olmo ML, Sastre J, et al. Zidovudine (AZT) causes an oxidation of mitochondrial DNA in mouse liver. Hepatology. 1999; 29: 985-987.

31. de la Asunción JG, Del Olmo ML, Gómez-Cambronero LG, et al. AZT induces oxidative damage to cardiac mitochondria: protective effect of vitamins $C$ and E. Life Sci. 2004; 76: 47-56.

32. Eck P, Erichsen HC, Taylor JG, et al. Comparison of the genomic structure and variation in the two human sodium-dependent vitamin $C$ transporters, SLC23A1 and SLC23A2. Hum Genet. 2004; 115: 285-294. 